\title{
Um Mecanismo de Compressão de Dados Local em Tempo Real Energeticamente Eficiente para Redes WBANs
}

\author{
Cainã Passos ${ }^{1}$, Aldri Santos ${ }^{1}$ (Orientador) \\ ${ }^{1}$ Núcleo de Redes Sem-Fio e Redes Avançadas (NR2) - PPGInf - UFPR \\ cpassoseinf.ufpr.br, aldri@inf.ufpr.br
}

\begin{abstract}
Among the networks for health care monitoring, we highlight wireless body networks (WBANs), which enable continuous and real-time monitoring of physiological signals. However, this monitoring results in the overuse of data transmission, drastically affecting the power consumption of the devices. This dissertation investigated these issues and has proposed the GROWN system, a hybrid approach to local data compression to reduce the redundancy of information in the data transmission and reduce the energy consumption of the devices, through WSN local data compression methods. GROWN was evaluated through experimentation and achieved a decrease in the energy consumption of the devices in face of transmitted signals and an increase in network lifetime.
\end{abstract}

Resumo. Dentre as redes computacionais voltadas ao cuidado com a saúde, destacam-se as redes corporais sem fio (WBANs), que possibilitam o monitoramento contínuo e em tempo real dos sinais fisiológicos. No entanto, esse monitoramento resulta em uso excessivo de transmissão de dados, afetando drasticamente o consumo de energia dos dispositivos. Esta pesquisa de dissertação investigou essas questões e propôs o sistema GROWN ${ }^{1}$, uma abordagem híbrida de compressão de dados local para reduzir a redundância de informações na transmissão de dados e redução do consumo de energia dos dispositivos, através de métodos de compressão de dados locais de WSN. O GROWN foi avaliado por meio de experimentação e obteve uma diminuição do consumo de energia dos dispositivos na transmissão dos sinais e um aumento na vida útil da rede.

\section{Introdução}

As redes corporais sem fio (Wireless Body Networks - WBANs) têm como propósito coletar, monitorar e transmitir sinais fisiológicos às diversas aplicações médicas e a profissionais de saúde, não interferindo na vida diária dos pacientes [Movassaghi et al. 2014]. As WBANs buscam oferecer um serviço de monitoramento contínuo e remoto, propiciando maior mobilidade e, em muitos casos, sendo desnecessária sua permanência num hospital. Por exemplo, numa situação de esgotamento dos hospitais, em caso de urgência de pandemia como o coronavirus, onde a ocupação de leitos hospitalares é uma questão sensível. Essas redes também dão suporte à telemedicina e a diagnósticos pró-ativos de modo a mitigar problemas de saúde dos pacientes. Os dispositivos numa WBAN caracterizam-se por sua heterogeneidade, variando as capacidades de memória, tarefas e tamanhos. Em geral, os dispositivos são energeticamente alimentados por pequenas baterias, adicionando mais restrições ao consumo de energia

\footnotetext{
${ }^{1}$ http://www.nr2.ufpr.br/ cpassos/grown.html (em inglês)
} 
na comunicação. A inoperância de um dispositivo por conta do consumo elevado de energia nas comunicações de aplicações de tempo real leva à perda de dados, e influencia no acompanhamento das condições de saúde do indivíduo. Logo, o consumo de energia é um grande desafio nas WBANs, visto que, os sensores necessitam de energia para detecção dos sinais e comunicação [Movassaghi et al. 2014].

Embora trabalhos como [Javaid et al. 2016, Khan and Pathan 2018] proponham protocolos de comunicação para maximizar o tempo de vida da rede, eles não consideram a redundância dos dados coletados e transmitem todas as informações. A fim de reduzir a redundância destacam-se as técnicas de compressão de dados, comumente dividida em duas classes: com perdas e sem perdas. Na primeira, uma quantidade aceitável da informação é descartada, tal que seja reduzida a quantidade de bits transmitidos. Já a segunda emprega tabelas de codificação para diminuir a quantidade de dados na transmissão e preservar a integridade do dado coletado. No entanto, há a necessidade do uso de ambas as classes nas redes WBANs, onde diversos dispositivos capturam sinais distintos. Nessas condições, elas atuam de maneira híbrida e a gestão do serviço de compressão precisa adaptar-se às características dos sinais de sensoriamento coletados, de modo a obter uma eficiência energética. Apesar dos trabalhos se adaptarem à heterogeneidade dos sinais, eles não levam em conta a latência máxima estabelecida por uma WBAN, que determina 125ms para aplicações médicas e 250ms para outras aplicações [Movassaghi et al. 2014].

\subsection{Objetivos e Contribuições}

Para atender tais desafios, investigou-se novas formas para reduzir o consumo de energia dos dispositivos sem afetar drasticamente a latência máxima permitida numa WBAN. Assim, esta dissertação buscou explorar e responder as seguintes questões de pesquisa atreladas ao problema levantado: $i$ ) Como o emprego de métodos de compressão de dados local contribuem para a eficiência energética dos dispositivos corporais nas WBANs? O emprego de métodos de compressão de dados local contribui para redução da informação transmitida. Além disso, é possível reduzir a redundância da informação coletada pelos sensores. Logo, a quantidade de pacotes transmitidos é reduzida e gerase eficiência energética nos dispositivos. $i i)$ Em que medida o uso de compressão de dados local interfere na transmissão das informações processadas pelos dispositivos corporais em aplicações de tempo real? As redes corporais sem fio de tempo real possuem uma latência máxima estabelecida conforme seu padrão [IEEE 2012]. O uso de compressão de dados local interfere no pós-processamento do sinal fisiológico coletado pelo sensor. Tendo em vista que toda informação leva um tempo para ser comprimida. Logo, a medida altera-se conforme o método empregado. iii) A aplicação de métodos de compressão de dados local auxilia para ampliar o tempo de vida dos dispositivos corporais numa WBAN? Abordagens clássicas transmitem toda informação coletada pelos sensores corporais. O emprego de métodos de compressão de dados local auxilia na redução de pacotes transmitidos. Assim, diminui-se o uso do rádio para transmissão, um dos principais consumidores de energia. Logo, aumenta-se o tempo de vida dos dispositivos.

O desenvolvimento dessa dissertação resultou em contribuições científicas na área de computação, com enfase em redes sem fio não estruturadas voltada à gerência de desempenho. A seguir, as contribuições deste trabalho estão descritas de forma detalhada: i) um estudo na literatura acerca dos métodos de compressão de dados local das redes de sensores sem fio que podem ser moldados para as redes corporais sem fio, além de méto- 
dos de compressão de dados local voltados especificamente para as redes WBANs. Esses métodos foram classificados de acordo com o tipo de rede pesquisado e sua aplicação para o problema de pesquisa; ii) Proposição do mecanismo GROWN: Ele baseia-se em métodos encontrados nas WSNs e analisa a diferença da correlação entre amostras consecutivas coletadas por sensores fisiológicos. Assim, a compressão desta diferença ocorre através de uma tabela fixa que não impacta drasticamente no atraso para apresentação da informação. Portanto, o mecanismo contribui para a redução no consumo de energia dos dispositivos; iii) Investigação experimental da eficácia do GROWN: Uma análise e discussão sobre os resultados obtidos pelo mecanismo para redução no consumo de energia dos dispositivos corporais, bem como sua aplicação em WBANs de tempo real.

\section{O Sistema GROWN para Compressão de Dados em WBANs}

O mecanismo GROWN objetiva reduzir a quantidade da informação transmitida de um dispositivo vestível para um dispositivo central (sink), que possui maior capacidade de processamento e energia sem afetar o atraso máximo de uma WBAN. O GROWN é executado sobre um conjunto de dispositivos vestíveis (nós) interligados numa rede WBAN conforme Figura 1. Esses nós possuem capacidade de processamento e de comunicação para coletar e disseminar sinais fisiológicos. Cada nó possui um identificador único $(I d)$, imutável no tempo, que o identifica na rede. Um dispositivo coleta um tipo de sinal fisiológico, processa e transmite a informação, através de um meio sem fio, para um dispositivo sink. Assume-se que há um controle de perdas de mensagens entre os dispositivos sensores e o sink através da tecnologia de comunicação sem fio disponível.

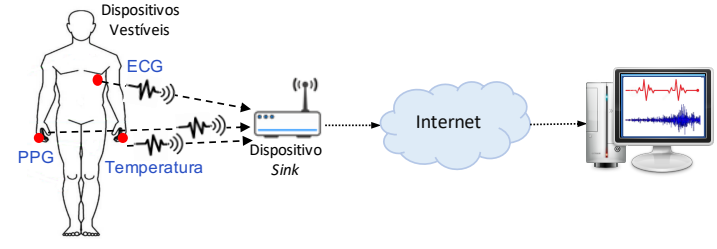

Figura 1. Modelo de Rede

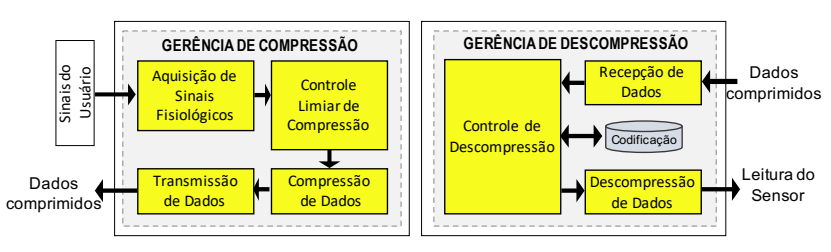

Figura 2. Arquitetura do GROWN

A arquitetura do GROWN, ilustrada na Figura 2, é composta pelo módulo Gerência de Compressão que recebe os sinais fisiológicos do usuário, realiza a sua compressão e envia ao módulo Gerência de Descompressão, responsável por recuperar o valor original da informação codificada através de um processo de descompressão.

O Módulo Gerência de Compressão obtém sinais fisiológicos do usuário, por meio de sensores vestíveis, e converte esses sinais analógicos para digital. Após análise, comprime esses dados e envia para um dispositivo sink. Ele é composto por componentes que convertem o sinal fisiológico analógico para digital; analisam o dado digital e definem a forma de compressão adequada; realizam a compressão dos dados; e transmitem esses dados ao dispositivo sink, por meio de uma conexão sem fio, para descompressão.

Os sensores vestíveis iniciam sua operação coletando sinais fisiológicos do usuário e, na medida que as leituras são realizadas, são recebidas pelo componente Aquisição de Sinais Fisiológicos. Esse componente converte o sinal fisiológico analógico para digital e o envia ao componente Controle Limiar de Compressão. Conforme descreve o Algoritmo 1, esse componente recebe a leitura atual do sensor (l.1). Se for a primeira 
leitura do sensor (l.3), armazena essa leitura para comparação com as próximas (l.4), registrando (l.6) e enviando essa leitura para compressão (l.14). Nas próximas leituras, ele verifica a diferença entre a leitura atual e a anterior (l.8), a fim de auxiliar a tomada de decisão sobre a compressão dessa informação. Para minimizar a redundância nas transmissões de informações, esse componente emprega um limiar previamente estabelecido. Ele verifica se a diferença absoluta entre as leituras atual e anterior é superior ao limiar estabelecido ou se não foi estabelecido um limiar (l.9). Nessas condições, ele verifica a diferença entre a leitura atual e anterior (l.10), armazena a leitura atual para comparação com as próximas (l.11) e a envia para compressão (l.14). Conforme ilustra a Figura 2, o componente Compressão de Dados recebe a leitura processada, comprime e encaminha os dados ao componente Transmissão. Este componente envia os dados comprimidos ao dispositivo sink, por meio da tecnologia sem fio disponível, à medida que eles chegam.

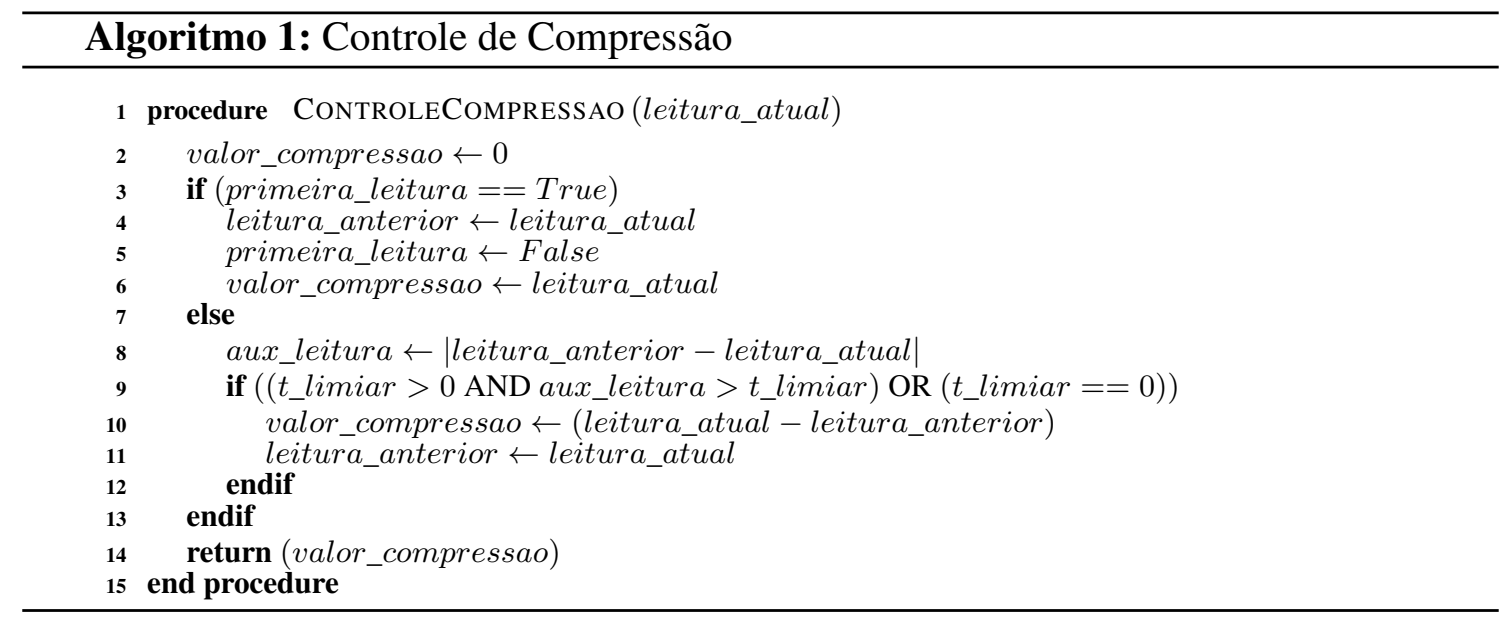

O componente Compressão de Dados codifica a informação recebida do componente Controle Limiar de Compressão $\left(e_{t s}\right)$ modificando a tabela de codificação Exponencial de Golomb (Exp-Golomb) de ordem $K=3$. As amostras recebidas $\left(e_{t s} \neq 0\right)$ são representadas por uma sequência de bits $b s_{i}$, que divide-se em duas partes $s_{i} \mid a_{i}$. A primeira parte $\left(s_{i}\right)$ identifica o grupo ao qual $e_{t s}$ pertence e ilustra o valor de $n_{i}$, que equivale à quantidade de bits necessária para representar $e_{t s}$. Assim, os primeiros $2^{k}-1$ valores de $n_{i}$ possuem grupos $s_{i}$ representados por $\left\lfloor\log _{2}\left(\left|e_{t s}\right|\right)\right\rfloor+1$, onde $s_{i}$ é representado por $k$ bits. Após este nível $\left(n_{i}>6\right)$, o valor do grupo $s_{i}$ é determinado por $\left\lfloor\log _{2}\left(\left|e_{t s}\right|\right)-1\right\rfloor$, onde os primeiros $s_{i}-1$ valores de $s_{i}$ são representados por 1 seguidos do valor 0 . A parte $a_{i}$ corresponde à representação binária de $e_{t s}$, que é um código de tamanho inteiro variável e gerado conforme as regras disponíveis na Tabela 1.

Tabela 1. Regras de compressão dos dados

\begin{tabular}{ll}
\hline Condição & Regra de compressão \\
\hline$e_{t s}<0$ & Efetuar complemento de 2 de $e_{t s}$, subtrair 1 e utilizar os $n_{i}$ bits menos significativos \\
$e_{t s}=0$ & Codificar $s_{i}$ como 000 e não representar $a_{i}$ \\
$e_{t s}>0$ & $a_{i}$ corresponde aos $n_{i}$ bits menos significativos do complemento de 2 de $e_{t s}$ \\
\hline
\end{tabular}

A Tabela 2 apresenta os valores de codificação sem perdas com os índices $n_{i}$, grupos $s_{i}$ e os valores de $e_{t s}$. Essa codificação depende da distribuição das diferenças entre os valores de $e_{t s}$. Diferenças mais frequentes associam-se a códigos mais curtos. Após 
a compressão de $e_{t s}$, uma nova informação é gerada e transmitida $\left(\operatorname{In} f_{t s}\right)$ ao dispositivo sink por meio da comunicação sem fio.

Tabela 2. Tabela de codificação proposta

\begin{tabular}{|c|c|c|c|c|c|c|c|c|c|}
\hline$n_{i}$ & $s_{i}$ & $e_{t s}$ & bit & byte & $n_{i}$ & $s_{i}$ & $e_{t s}$ & bit & byte \\
\hline 0 & 000 & 0 & 3 & 1 & 4 & 100 & $-15 \ldots \ldots-8,+8 \ldots \ldots+15$ & 7 & 1 \\
\hline 1 & 001 & $-1,+1$ & 4 & 1 & 5 & 101 & $-31 \ldots-16,+16 \ldots+31$ & 8 & 1 \\
\hline 2 & 010 & $-3,-2,+2,+3$ & 5 & 1 & 6 & 110 & $-63, \ldots,-32,+32, \ldots,+63$ & 9 & 2 \\
\hline 3 & 011 & $-7, \ldots,-4,+4, \ldots,+7$ & 6 & 1 & 7 & 11110 & $-127, \ldots,-64,+64, \ldots,+127$ & 12 & 2 \\
\hline
\end{tabular}

O Módulo Gerência de Descompressão opera no dispositivo sink, que detém recursos de energia, armazenamento e processamento para executar a descompressão de dados enviados por dispositivos vestíveis pertencentes à WBAN. O componente Recepção de Dados (RD) inicia a recuperação do valor correspondente ao sinal fisiológico coletado e enviado pelos dispositivos vestíveis. Após receber a mensagem com os dados comprimidos $\left(b s_{i}\right)$ e a identificação do dispositivo de origem $\left(D_{I d}\right)$, ele separa essas informações para uso posterior. O Controle de Descompressão $(\mathrm{CD})$ recebe estas informações de RD, e como descrito no Algoritmo 2, mantém uma Lista de Referências (LR), com a identificação $\left(D_{I d}\right)$ dos dispositivos vestíveis conectados à rede e o valor da última leitura $\left(\hat{x}_{t s}\right)$.

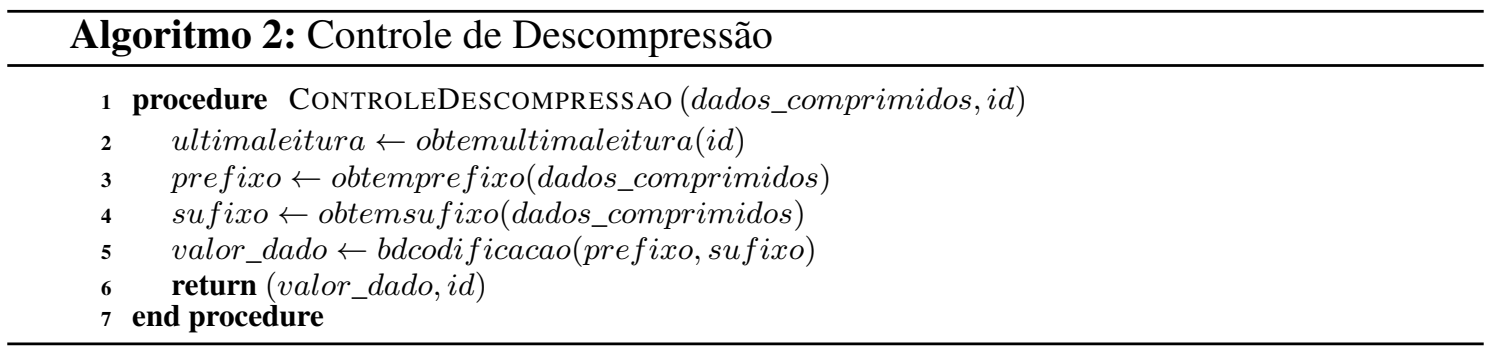

Após CD consultar a LR com o $D_{I d}$ ele obtém o $\hat{x}_{t s}$ desse dispositivo, o prefixo $\left(s_{i}\right)$ e o sufixo para o $b s_{i}$ recebido (l.2-4). Em seguida, ele consulta a base de dados Codificação (l.5), que armazena os índices $n_{i}$, os grupos $s_{i}$ e seus valores $a_{i}$. Cada valor de $a_{i}$ é exclusivo em todo grupo $s_{i}$, permitindo a recuperação da informação por meio de poucas instruções, adequando-se a uma operação em tempo real. Depois, CD obtém o valor correspondente da informação $b s_{i}$, adquire a última leitura do dispositivo $D_{I d}$ salva na Lista de Referências $\left(\hat{x}_{t s}\right)$, atualiza a LR e encaminha os valores ao componente Descompressão de Dados. Este componente calcula a diferença entre $\hat{x}_{t s}$ antes da atualização da LR e o valor obtido de $b s_{i}$. Por fim, o valor lido pelo sensor é disponibilizado para uso.

\subsection{Funcionamento}

Considere um ambiente de WBAN com um dispositivo vestível de sensoriamento contínuo de temperatura e que este dispositivo interage com um smartphone atuando como o gateway através da tecnologia bluetooth, como ilustra a Figura 3. O dispositivo vestível obtém o sinal fisiológico de temperatura da pessoa e converte de analógico para digital em uma representação binária $\left(x_{t s}\right)$ com $R$ bits. Tendo em conta que o valor lido foi 38, então $x_{t s}$ em 8 bits torna-se 00100110. A tomada de decisão sobre a compressão do dado depende do filtro de predição $(T)$, que é pré-definido. Assumindo $T=1$, esse filtro verifica o valor de $x_{t s}$, e como trata-se da primeira leitura do sensor, a leitura anterior $\left(\tilde{x}_{t s}\right)$ recebe o valor 0 (zero). Dessa forma, o erro de predição $\left(e_{t s}\right)$, que corresponde à diferença entre leituras sucessivas, é $e_{t s}=x_{t s}-\hat{x}_{t s}=38$. 


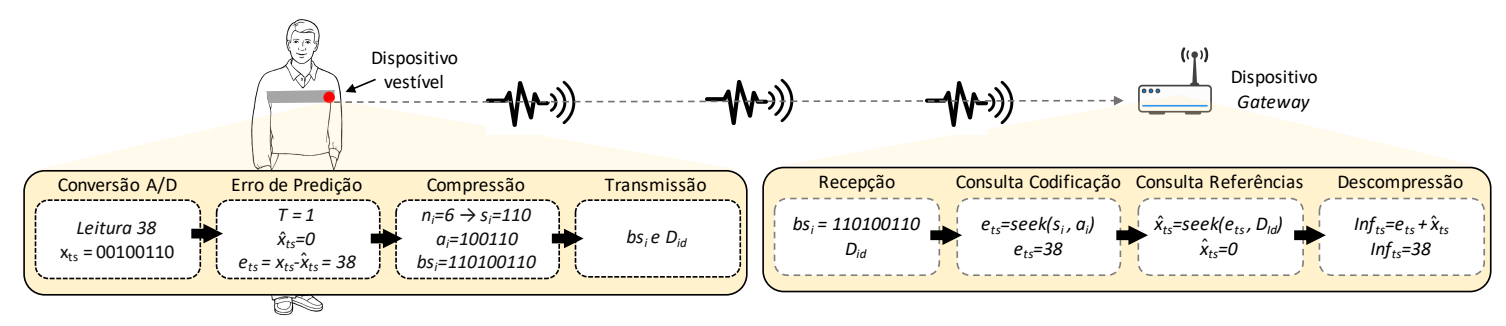

Figura 3. Exemplo de funcionamento

O valor de $e_{t s}$ é convertido em uma sequência de bits $\left(b s_{i}\right)$ composta de duas partes $s_{i} \mid a_{i} . s_{i}$ representa o grupo $\left(n_{i}\right)$ ao qual $e_{t s}$ pertence. Assim, $n_{i}=\left\lfloor\log _{2}(|38|)\right\rfloor+1=6$. Consultando-se a tabela dos valores de codificação (Tabela 2), verifica-se que $s_{i}$ pertence ao grupo 110. Em seguida, obtém-se $a_{i}$ convertendo para binário de $e_{t s}=38$, resultando em $a_{i}=100110$. Logo, $b s_{i}=110100110$. Então a mensagem transmitida ao gateway é composta da $b s_{i}$ e da identificação do dispositivo vestível $\left(D_{I d}\right)$. Já o gateway ao receber a mensagem do dispositivo vestível verifica sua identificação $\left(D_{I d}\right)$ e os dados recebidos $\left(b s_{i}\right)$. Os dados são decompostos, obtendo-se $s_{i}=110$ e $a_{i}=100110$. Consultandose a Tabela de Codificação com $s_{i}$ e $a_{i}$, obtém-se o erro de predição $\left(e_{t s}\right)$. Em seguida, uma consulta à Tabela de Referências usando $e_{t s}$ e $D_{I d}$ retorna o último valor recebido desse dispositivo, $\hat{x}_{t s}=0$. Finalmente, a informação $\left(\operatorname{In} f_{t s}\right)$ correspondente ao sinal do dispositivo vestível é recuperada, $\operatorname{In} f_{t s}=e_{t s}+\hat{x}_{t s}=38$.

\section{Metodologia da Avaliação e Resultados}

O módulo gerência de descompressão, localizado no sink, foi implementado no Android Studio, versão 3.6.1. O aplicativo desenvolvido ${ }^{2}$ foi instalado em um celular Motorola, modelo Moto $\mathrm{G}^{4}$ Plus, que opera com o sistema operacional Android, versão 7.0. A implementação do módulo gerência de compressão com e sem perdas ocorreu através da linguagem C++ e compilada para placas Arduino com características da Tabela 3.

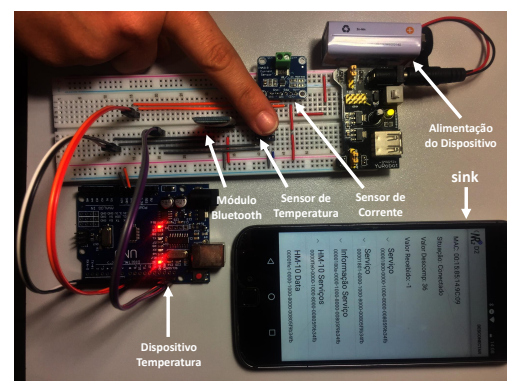

Tabela 3. Dispositivos corporais

\begin{tabular}{lccc}
\hline \multirow{2}{*}{ Característica } & \multicolumn{3}{c}{ Dispositivo } \\
\cline { 2 - 4 } & Temperatura & PPG & ECG e ECGBD \\
\hline Placa & Arduino UNO & Arduino UNO & Arduino Mega \\
Microcontrolador & ATmega328P & ATmega328P & ATmega2560 \\
Sensor Corporal & MLX90614 & PulseSensor & AD8232 \\
\hline
\end{tabular}

Figura 4. Testbed Temperatura

O ambiente de experimentação consistiu de três dispositivos corporais fixos e um dispositivo celular atuando como sink da WBAN, conforme ilustrado na Figura 1. Esses dispositivos corporais correspondem ao sensoriamento de Temperatura, ECG e PPG (Tabela 3). Para aprimorar a análise do GROWN, criou-se um quarto dispositivo (ECGBD) para comprimir sinais ECG da base de dados (MIT-BIH Arrhythmia ${ }^{3}$ ) salva em um módulo SD. Os dispositivos foram produzidos em Protoboards Mb102. As protoboards

\footnotetext{
${ }^{2}$ Código disponível em https://github.com/cainapassos/GROWN

${ }^{3}$ https://alpha.physionet.org/content/mitdb/1.0.0/
} 
fornecem energia para todos os sensores e placas Arduino, responsáveis pela gerência dos sensores e do GROWN na compressão. Elas são alimentadas por fontes reguladas para operar em $5 \mathrm{~V}$. Cada fonte é alimentada por uma bateria de $9 \mathrm{~V}$ com capacidade energética de $400 \mathrm{mAh}$. Cada dispositivo coleta apenas um tipo de sinal fisiológico.

A compressão e a descompressão dos dados impõem um processamento adicional ao sensor e ao dispositivo sink. As variações da compressão (AC) e descompressão (AD) ao longo do tempo são apresentadas na Figura 5. As variações na AC são devidas à correlação entre amostras consecutivas, que quanto maior, menor é a diferença. Assim, o GROWN gera códigos mais curtos para compressão e diminui o atraso total. Esta correlação é observada no sinal de temperatura, que apresentou um atraso médio de compressão de $\approx 1 \mathrm{~ms}$, visto no Gráfico 5(g), visto que ele sofre poucas alterações ao longo do tempo.
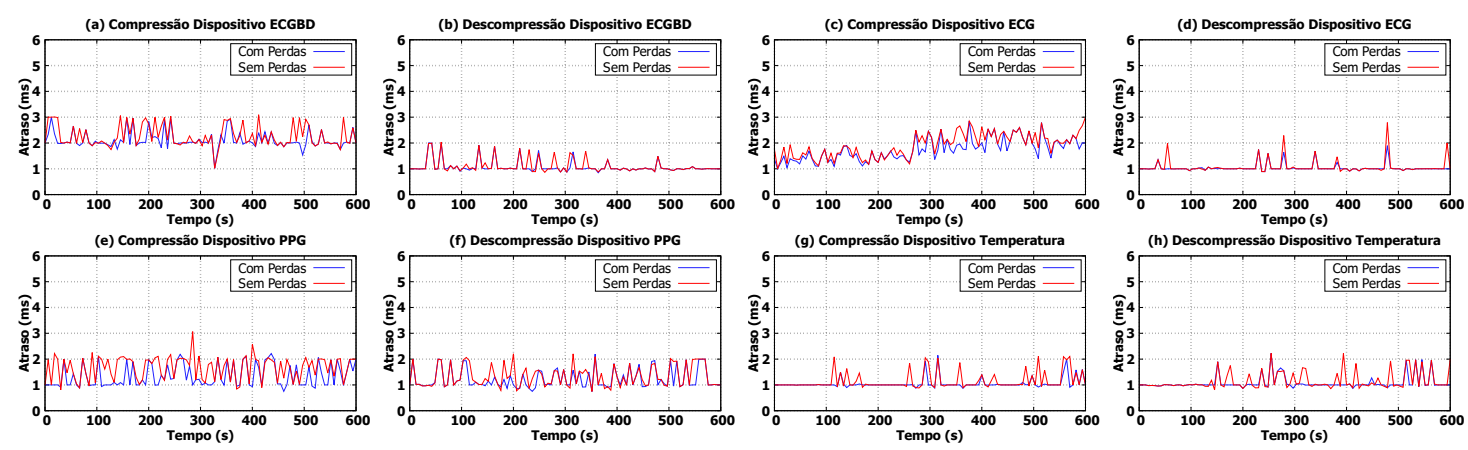

Figura 5. AC e AD alcançado pelo GROWN nos sinais sensoriados

A correlação dos demais sinais apresentou uma grande variação ao longo do tempo, como visto nos Gráficos 5(a), 5(c) e 5(e). Enquanto o dispositivo ECG apresentou um atraso médio de $\approx 3 \mathrm{~ms}$, o atraso do PPG foi de $\approx 2 \mathrm{~ms}$. O GROWN obteve um comportamento similar com a base de dados real no dispositivo ECGBD, com média de $\approx 3 \mathrm{~ms}$ na compressão. Este comportamento repete-se no dispositivo sink. Porém, devido ao seu maior poder computacional, a descompressão impõe um atraso menor, $\approx 1 \mathrm{~ms}$, como exibidos nos Gráficos 5(b), 5(d) e 5(h).

O Atraso médio (AM) deve-se a compressão, transmissão e descompressão do GROWN conforme Figura 6. A transmissão dos dados impôs um atraso médio de $\approx 49 \mathrm{~ms}$ a todos os dispositivos, assim como [Gatouillat et al. 2018], que verificou um intervalo entre amostras de $\approx$ $48,75 \mathrm{~ms}$ em conexões Bluetooth BLE no Android 7.0. Em geral, o GROWN aumentou em 7,84\% o tempo total de transmissão aos dispositivos ECG e ECGBD, $6 \%$ ao PPG, e 2,04\% para o de Temperatura. Assim, o tempo de compressão e descompressão não afeta drasticamente o atraso entre amostras consecutivas e demonstra a aplicabilidade do GROWN nas WBANs de tempo real. O uso do GROWN
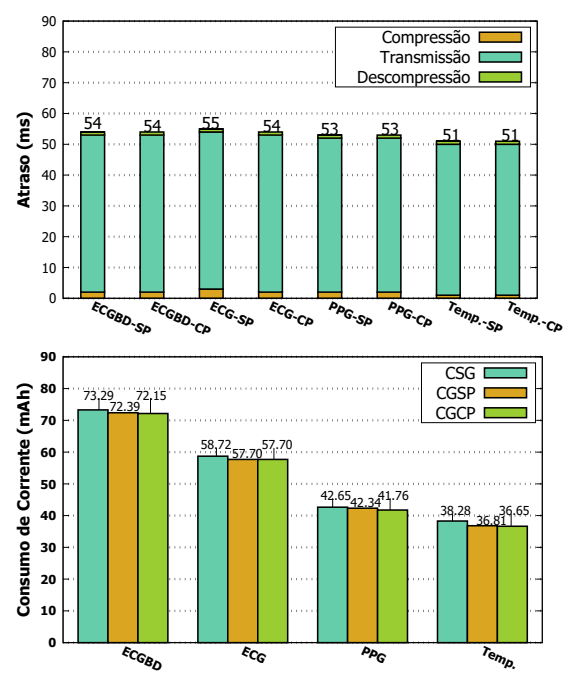

Figura 6. AM e CED alcançados pelo GROWN 
com perdas (CGCP) e sem perdas (CGSP) reduziu o consumo de energia dos dispositivos (CED) sobre os dispositivos sem o GROWN (CSG) conforme Figura 6. A redução aumenta do tempo de vida dos dispositivos, como visto na Tabela 4. Para mitigar o impacto do módulo de comunicação sem fio que permanece ativo e emparelhado nas experimentações, o dispositivo Temperatura foi configurado para desativar a comunicação sempre que duas amostras consecutivas não são transmitidas. Essa configuração aumentou a vida útil do dispositivo em 53,73\% com CGCP e 48,37\% com CGSP, como ilustra a Tabela 5.

Tabela 4. Tempo de vida

\begin{tabular}{lccc}
\hline \multirow{2}{*}{ Dispositivo } & \multicolumn{3}{c}{ Autonomia da bateria $(h)$} \\
\cline { 2 - 4 } & CSG & CGSP & CGCP \\
\hline ECGBD & 5,46 & 5,53 & 5,54 \\
ECG & 6,81 & 6,93 & 6,93 \\
PPG & 9,38 & 9,45 & 9,58 \\
Temperatura & 10,45 & 10,87 & $\mathbf{1 0 , 9 1}$ \\
\hline
\end{tabular}

Tabela 5. (CED) Temperatura

\begin{tabular}{lcc}
\hline \multirow{2}{*}{ Compressão } & \multicolumn{2}{c}{ Autonomia da bateria $(h)$} \\
\cline { 2 - 3 } & Normal & Sleep \\
\hline CGCP & 10,91 & $\mathbf{1 6 , 0 5}$ \\
CGSP & 10,87 & $\mathbf{1 5 , 5 0}$ \\
\hline
\end{tabular}

\section{Conclusão e Impacto da Dissertação}

Este artigo resumiu a pesquisa de dissertação que investigou um modo de otimizar o tempo de vida dos dispositivos nas redes WBANs. Essas redes serão essenciais ao suporte à telemedicina e também diagnósticos pró-ativos de modo a mitigar problemas de saúde de pacientes remotos em áreas urbanas e rurais. Assim, desenvolveu-se o GROWN, um mecanismo para gerenciamento da compressão de dados local visando reduzir a redundância da informação na transmissão de sinais. Experimentações avaliaram a eficácia do GROWN e os resultados demonstram sua capacidade de gerenciar a compressão e descompressão dos dados sensoriados. Uma avaliação experimental demonstrou que essa abordagem propiciou a diminuição do consumo de energia dos dispositivos e o aumento do tempo de vida da rede sem exceder o limite da latência. Esta pesquisa também colabora para melhorar o desempenho da comunicação em áreas como realidade aumentada, jogos massivos, entre outras. As contribuições deste trabalho resultaram nas publicações [Passos et al. 2020b, Passos et al. 2020a] e o prêmio de melhor artigo do XXV WGRS (Workshop de Gerência e Operação de Redes e Serviços).

\section{Referências}

Gatouillat, A., Massot, B., Badr, Y., Sejdić, E., and Gehin, C. (2018). Building iot-enabled wearable medical devices: an application to a wearable, multiparametric, cardiorespiratory sensor.

IEEE (2012). Ieee standard for local and metropolitan area networks - part 15.6: Wireless body area networks. IEEE Std 802.15.6-2012, pages 1-271.

Javaid, N., Ahmad, A., Tauqir, A., Imran, M., Guizani, M., Khan, Z. A., and Qasim, U. (2016). Modeling induction and routing to monitor hospitalized patients in multi-hop mobility-aware body area sensor networks. EURASIP Journal on Wireless Comm. and Networking, 2016(1):147.

Khan, R. A. and Pathan, A.-S. K. (2018). The state-of-the-art wireless body area sensor networks: A survey. International Journal of Distributed Sensor Networks, 14(4):1550147718768994.

Movassaghi, S., Abolhasan, M., Lipman, J., Smith, D., and Jamalipour, A. (2014). Wireless body area networks: A survey. IEEE Communications Surveys \& Tutorials, 16(3):1658-1686.

Passos, C., Junior, C. P., Batista, A., de Lima, M., and dos Santos, A. (2020a). Compressão de dados local em tempo real energeticamente eficiente para redes wban. In Anais do XXV Workshop de Gerência e Operação de Redes e Serviços, pages 85-98. SBC.

Passos, C., Pedroso, C., Batista, A., Nogueira, M., and Santos, A. (2020b). Grown: Local data compression in real-time to support energy efficiency in wban. In 2020 IEEE Latin-American Conference on Communications (LATINCOM), pages 1-6. IEEE. 